\title{
THEORETICAL AND EXPERIMENTAL ASPECTS OF FRACTIONATION MECHANISMS IN LIQUID CHROMATOGRAPHY ON MACROMOLECULAR STATIONARY PHASES (GEL OR BONDED CHAINS)
}

\author{
Jacqueline Lecourtier; Roland Audebert and Claude Quivoron \\ Laboratoire de Physico-Chimie Macromoléculaire, Université Pierre et Marie \\ Curie, E.S.P.C.I., 10, rue Vauquelin, 75231 Paris Cedex 05, France
}

\begin{abstract}
In a preliminary part, we deal with the partition mechanism of low or high molecular weight solutes, by selective dissolution in swollen gels. After summing up the theoretical expressions, we present some experimental evidences for a fractionation mechanism based on such a phenomenon, which may interfere, in many cases, with the classical steric exclusion effect in GPC. Most of this paper is devoted to chemically bonded stationary phases which gained increasing importance in H.P.L.C., during the past few years. Owing to our previous works on gels, we theoretically studied the partition coefficient of low or high molecular weight solutes between a mobile phase and a stationary phase, constituted by macromolecular chains bonded to an inert surface. Variations of partition coefficient with the molecular sizes of solute, solvent and graft, and with the molecular interaction parameters between these species, are presented. In a complementary aspect, we describe the recent preparation of model chemically bonded phases of silica/polyethyleneoxide and silica/polystyrene types. The retention data obtained for various solutes on these bonded phases are discussed in terms of silica loading, grafting chain length, solute size and solvent nature. These theoretical and experimental studies lead to practical consequences concerning the relative parts of the dissolution effect into the grafted phase, the adsorption on the mineral support and the steric exclusion effect due to the residual porosity.
\end{abstract}

\section{INTRODUCTION}

Cross-linked organic gels are now classical materials which application is well known, for instance, in the field of elastomers, plastics and biomaterials. The case of macromolecular chains chemically bonded onto a surface by one of their ends is more recent. Various applications are already suggested : compatibilization of prosthesis with blood, supported catalysis, stabilization of polymer emulsions, selective adsorption of hydrocarbon polluents, etc...

However, there is a field of application common to gel and grafted materials, in increasing importance : the use as stationary phases in liquid chromatography. Organic porous gels are thus used for many years in Gel Permeation Chromatography (GPC) for the fractionation of macromolecules (Ref.1-2) and more recently for the separation of low molecular weight solutes (Ref.2-5). In another hand, chemically bonded stationary phases are commonly used in liquid liquid chromatography (LLC) (Ref.5-11) ; some of them having macromolecular grafts. Besides, grafted GPC packings were proposed for the molecular weight characterization of organic or hydrosoluble polymers and some of them have been commercialized, such as "glycophases" (Ref.12-14) or " $\mu$-bondage1" (Ref.15-16).

The aim of this lecture is to present some theoretical and experimental aspects of the mechanisms involved in GPC or LLC separations on these macromolecular stationary phases. As very few studies have been devoted until now to provide information in the field of macromolecular bonded phases, stress will be laid on that kind of stationary phases.

\section{ORGANIC GEL STATIONARY PHASES}

Several types of mechanisms were proposed to explain the polymer fractionations observed in GPC experiments and were discussed in reviews recently published (Ref.2,17). Under usual chromatographic conditions, it is well accepted now that GPC fractionations involve an equilibrium of solutes between the mobile and gel phases, as illustrated Fig. 1.

Mechanism (a) corresponds to the classical steric exclusion effect in the pores, which is unanimously assumed to be the main one in polymer chain fractionations by GPC. However, some recent experimental results (Ref.18-28) oblige to consider the interference of two non-exclu- 
sion mechanisms which cause deviations from the universal calibration curve (Ref.29). Adsorption mechanism (b), discussed in Professors Dawkins' and Belenkij's lectures, may be especially important in the case of rigid or semi-rigid packings with large specific surface areas, but it is not probably predominant for swollen gels.

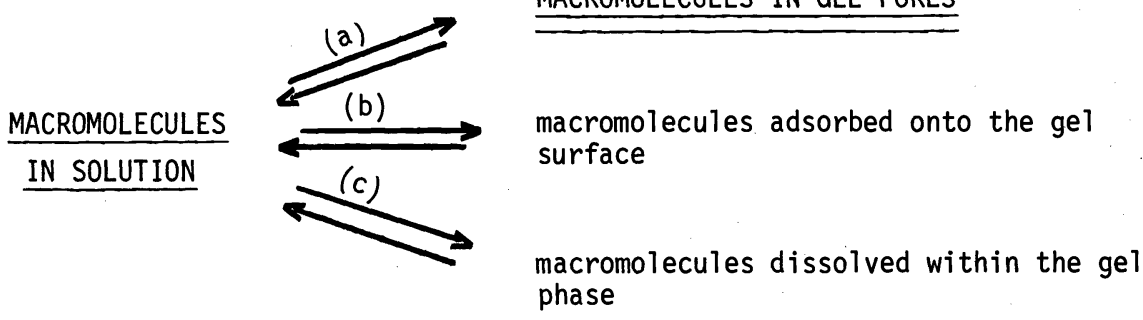

Fig. 1 Different mechanisms supposed to be independent, involved in fractionation on swelling gels.

We would consider here mechanism (c) which arises from a solute dissolution within a non rigid gel. In other words, when a swelling gel is mixed with a solution, its macromolecular network is swollen both by the solvent and the solute and it acts as a LLC stationary phase.

For that last mechanism alone, the elution volume of a solute is given by :

$$
v_{e}=V_{0}^{\prime}+K_{D} \cdot v_{S}
$$

where $V^{\prime}$ is the elution volume of solutes which do not penetrate into the swollen gel volume $V_{S} . K_{D}^{0}$ is the partition coefficient of the solute between the mobile and the gel phases:

$$
K_{D}=\frac{\text { solute concentration in the gel phase }}{\text { solute concentration in the mobile phase }}
$$

That dissolution mechanism was particularly studied in our laboratory by theoretical and experimental approaches (Ref. 30-32) and results obtained were confronted with some other experimental data (Ref.33-36). We will not mention here our thermodynamical calculation of $K_{0}$ (Ref. 30 ), based on Flory's theory for gels. It consists in evaluating the free energy changes $\Delta G$ of the solute/solvent and solute/solvent/gel mixtures, in order to equal the two expressions for chemical potential of the solute in the two phases. These calculations lead to :

$$
\log K_{D}=-\frac{r_{2}}{r_{1}} \phi_{3}-\left(\phi_{3}^{1 / 3}-\frac{\phi_{3}}{2}\right) \cdot \frac{r_{2}}{x_{3}}+x_{12} \cdot \frac{r_{2}}{r_{1}} \cdot \phi_{3}+x_{13} \cdot \frac{r_{2}}{r_{1}} \cdot \phi_{3}\left(1-\phi_{3}\right)-x_{23} r_{2} \phi_{3}
$$

$$
\text { (1: solvent ; } 2 \text { : solute ; } 3: \text { gel) }
$$

$r_{1}$ : segment number of each species ; $x_{j j}$ : Flory's interaction parameter ; $\phi_{3}:$ volume fractton of the swollen gel; $x_{3}$ : segment number average of units between two cross-1inked points, assuming a perfect gel. (For calculations, $r_{1}$ is taken as 1 in all this paper).

The swelling of the gel expressed by its volume fraction $\phi_{3}$, is a function of $x_{13}$ and $x_{3}$ :

$$
\log \left(1-\phi_{3}\right)+\phi_{3}+x_{13} \phi_{3}^{2}+\left(\phi_{3}^{1 / 3}-\frac{\phi_{3}}{2}\right) \cdot \frac{r_{1}}{x_{3}}=0
$$

The expression of $K_{D}$ allows to study the partition mechanism of solutes by selective dissolution according to their molecular size $\left(r_{2}\right)$ and to predict the influence of the swollen gel structure $\left(\phi_{3}\right.$ or $\left.x_{3}\right)$, the solvent nature $\left(x_{12}\right.$ and $\left.x_{13}\right)$ and the gel/solute interaction $\left(x_{23}\right)$. Fig. 2 gives logarithm of solute molecular weights $M_{2}$ versus $K_{D}$, for various values of the gel structure parameter $x_{3}$ and when there is no enthalpic effect $\left(x_{i j}=0\right)$.

It appears that $\mathrm{K}_{\mathrm{D}}$ varies between 0 and 1 , when the solute molecular weight decreases, which illustrates a fractionation based only on an entropic effect. This fractionation by dissolution becomes more important for high molecular weights as the cross-linked degree of the swollen gel becomes obviously lower. Curves are similar when $x_{23}=0$ and for a solvent having the same affinity towards the gel and solutes $\left(\chi_{12}=\chi_{13} \neq 0\right)$.

Experimental results obtained in our laboratory and some others experimental data are in good agreement, at least qualitatively, with these predictions. First of a11, Benoit, Rempp and al (Ref.33-34) studied tailor-made gels by copolymerization of divinyl-benzene with bianionic living polystyrenes of various macromolecular weights. They obtained swollen gels, not macro- 
porous in which the length between two points of cross-linking is determined by the molecular weight of the precursor polystyrene. Elutions of polystyrene standards in tetrahydrofuran (THF) give curves Log $M_{2}$ versus $K_{D}$, plotted in Fig. 3 and which are quite similar with those predicted theoretically. Particularly, gels are more efficient towards high molecular weight solutes as their degrees of cross-linking decrease. A recent work confirms such a macromolecular solute behavior towards swelling gels (Ref.35).

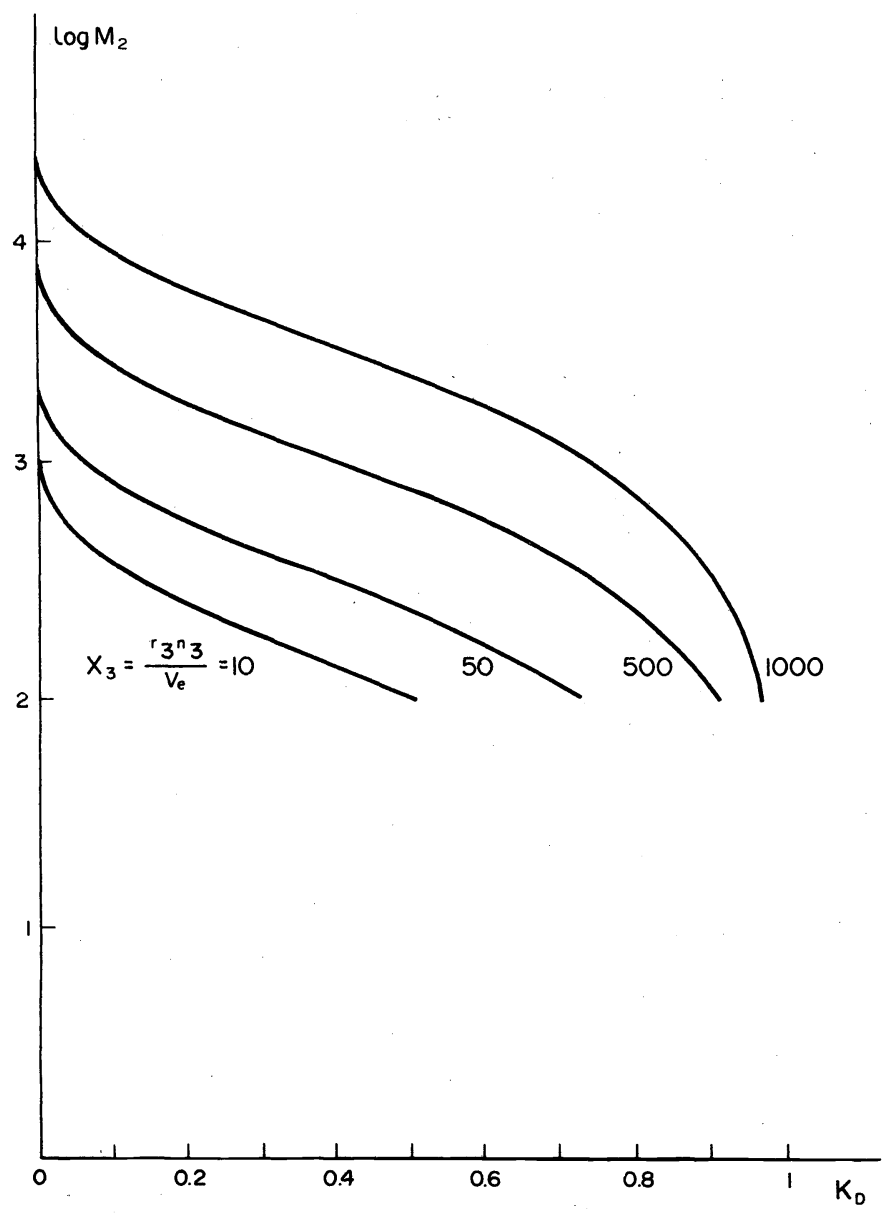

Fig. 2 Log $M_{2}$ versus $K_{D}$ (partition coefficient) for various values of $x_{3}=r_{3} n_{3} / \nu{ }^{\prime}\left(n_{3}\right.$ is the number of polymer chains, the statistical reticulation of which gave the gel and $v_{0}$ is the total number of cross-linked units which constitute the gel). Curves are plotted with $M_{2}=10^{2} \cdot r_{2}$ and $x_{13}=x_{12}=x_{23}=0$.

A second series of experimental evidences was obtained in our laboratory with triazinic gels (Ref. 31-32), prepared by polycondensation of diamines with dichlorotriazine $\left(\mathrm{TCl}_{2}\right) / \mathrm{Cyanuric}$ chloride $\left(\mathrm{TCl}_{3}\right.$ ) mixtures (see Fig.4). Different degrees of cross-linking were determined by varying the ratio $\gamma=\mathrm{TCl}_{3} /\left(\mathrm{TCl}_{2}+\mathrm{TCl}_{3}\right)$. The gel swelling is then a function of $\gamma$ and $n$ (length of each diamine used).

Mercury intrusion experiments showed that these swollen gels have mainly great size pores and accordingly should be efficient only in the high molecular weight range, by a GPC effect. Fig. 5 gives the general shape of calibration çurves which exhibit two parts, where $V$ '。 corresponds to molecular weights varying from $10^{3}$ to $10^{4}$.

The first part of the curve was explained by the classical steric exclusion effect, consistent with the great size of pores. As the gel porosity study does not indicate pores of small size, the fractionation observed for molecular weights lower than $M^{\prime}$, was attributed to the solute dissolution within the gel $\left(V_{e}=V^{\prime}{ }_{0}+K_{D} \cdot V_{s}\right)$.

Fig. 6 gives calibration curves of polystyrene and $n$-alkane samples in benzene, for various values of the degree of cross-linking $\gamma$. Similar variations are obtained with gel of the same degree of cross-linking but with various lengths of diamine chain. These experimental results are in good agreement with the theoretical one, since gels are more efficient towards 
high molecular weights as their swelling increases.

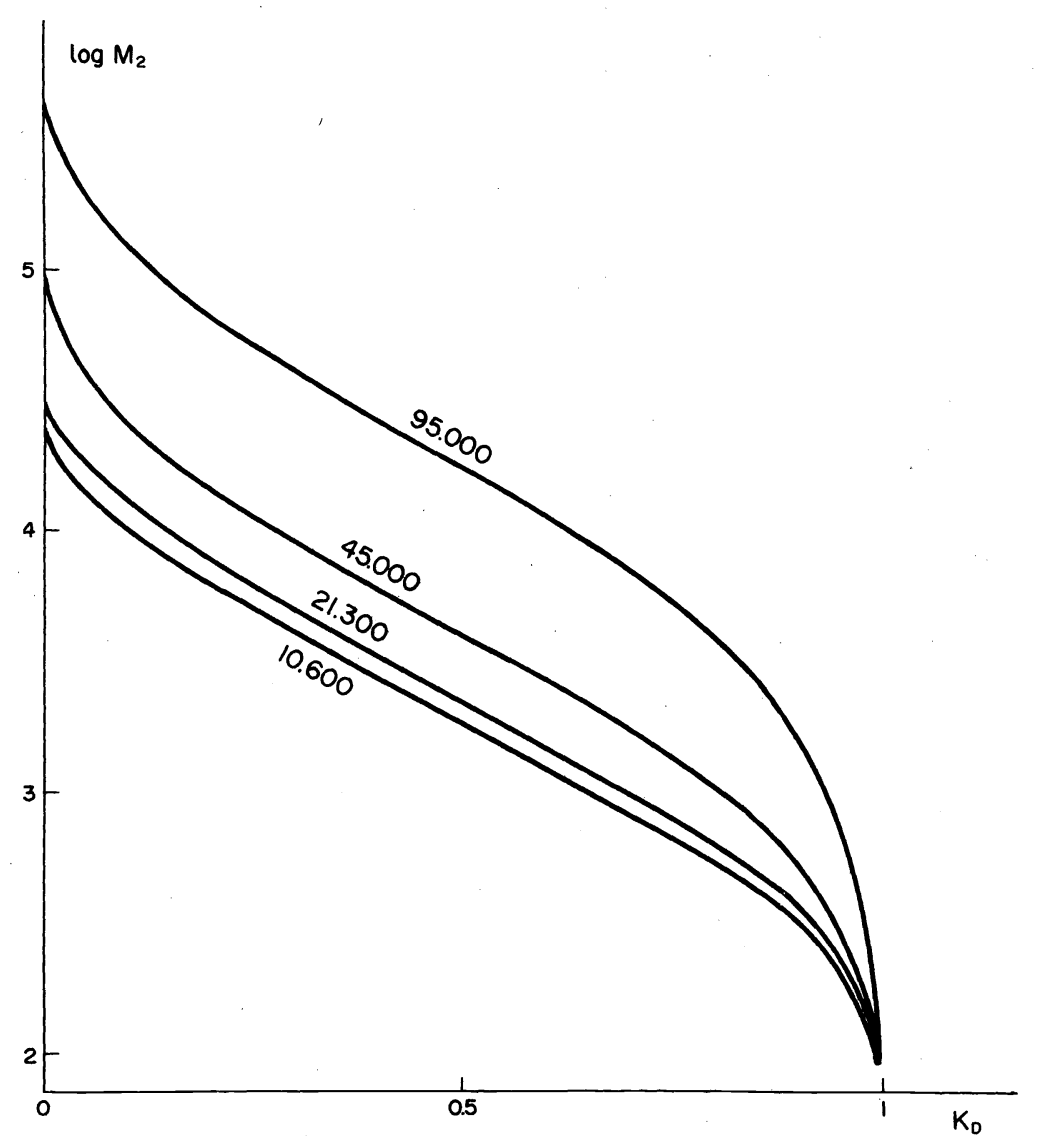

Fig. 3 Experimental fractionation of polystyrene solutes obtained on 4 non porous styrene - divinylbenzene model gels with different chain lengths between cross-linking points (Ref.33-34).

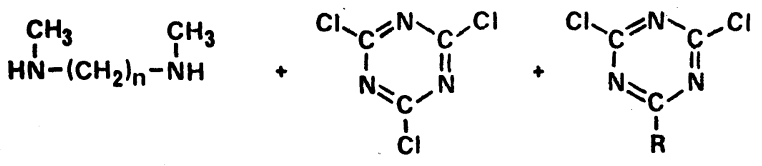

$$
\begin{aligned}
& \downarrow-\mathrm{HCl}
\end{aligned}
$$

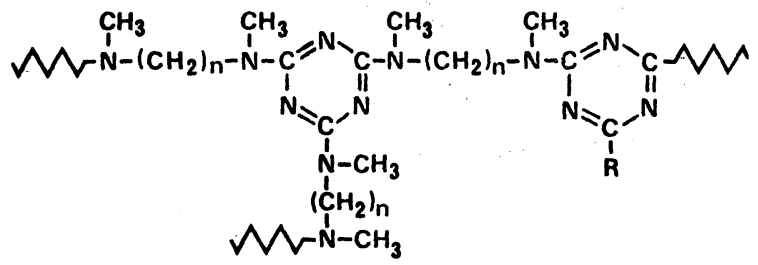

Fig. 4 Molecular structure of triazinic model gels used in GPC experiments. 


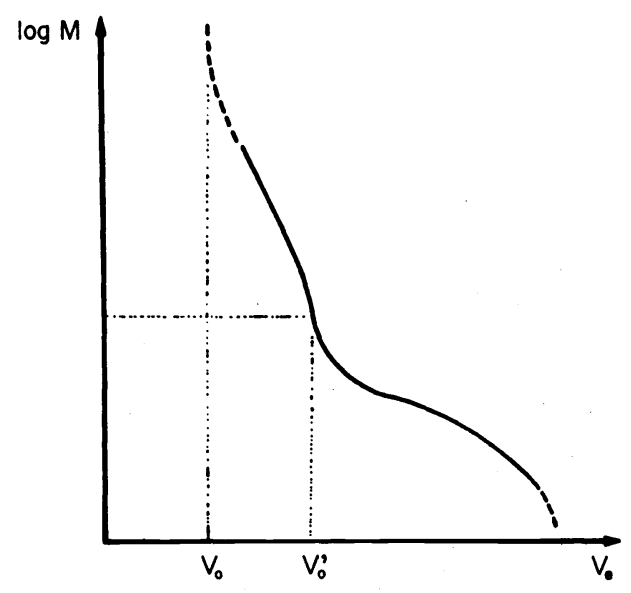

Fig. 5 General shape of calibration curves obtained with the triazinic model gels.

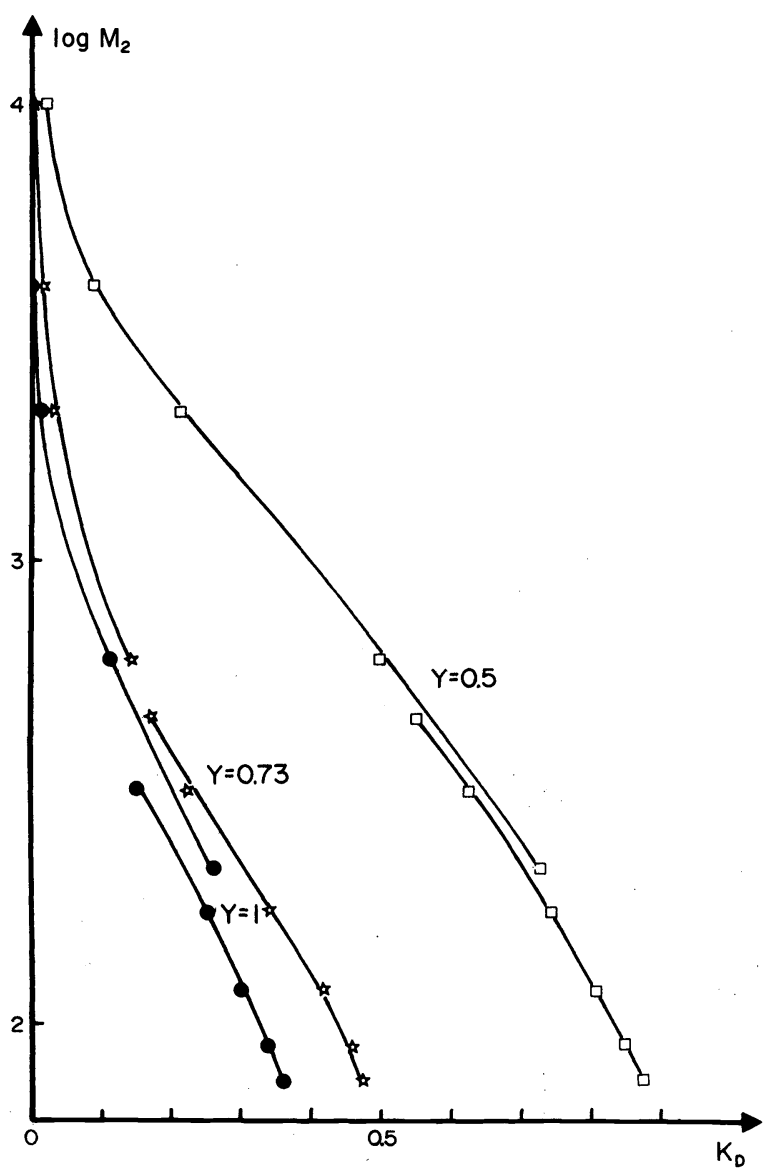

Fig. 6 Variation of the curve Log $M_{2}$ versus $K_{0}$ with the degree of cross-1inking $\left(\gamma=0,5 ; \gamma=0,73\right.$ and $\left.{ }^{2} \gamma=1\right)$. Gel : hexamethylened iamine $\mathrm{TCl}_{3} / \mathrm{TCl}_{2}(\mathrm{R}=$ morpholinyl) ; Solvent : benzene ; Solutes : polystyrene (high molecular weight range) and n-alkanes (low molecular weight range).

Another aspect of the solute dissolution mechanism concerns the influence of the gel/solute interactions. Fig. 7 shows, for a given solvent/gel system, the evolution of the theoretical curves $\log M_{2}=f\left(K_{D}\right)$ with the gel/solute interaction parameters $x_{23}$ (Ref.30). 


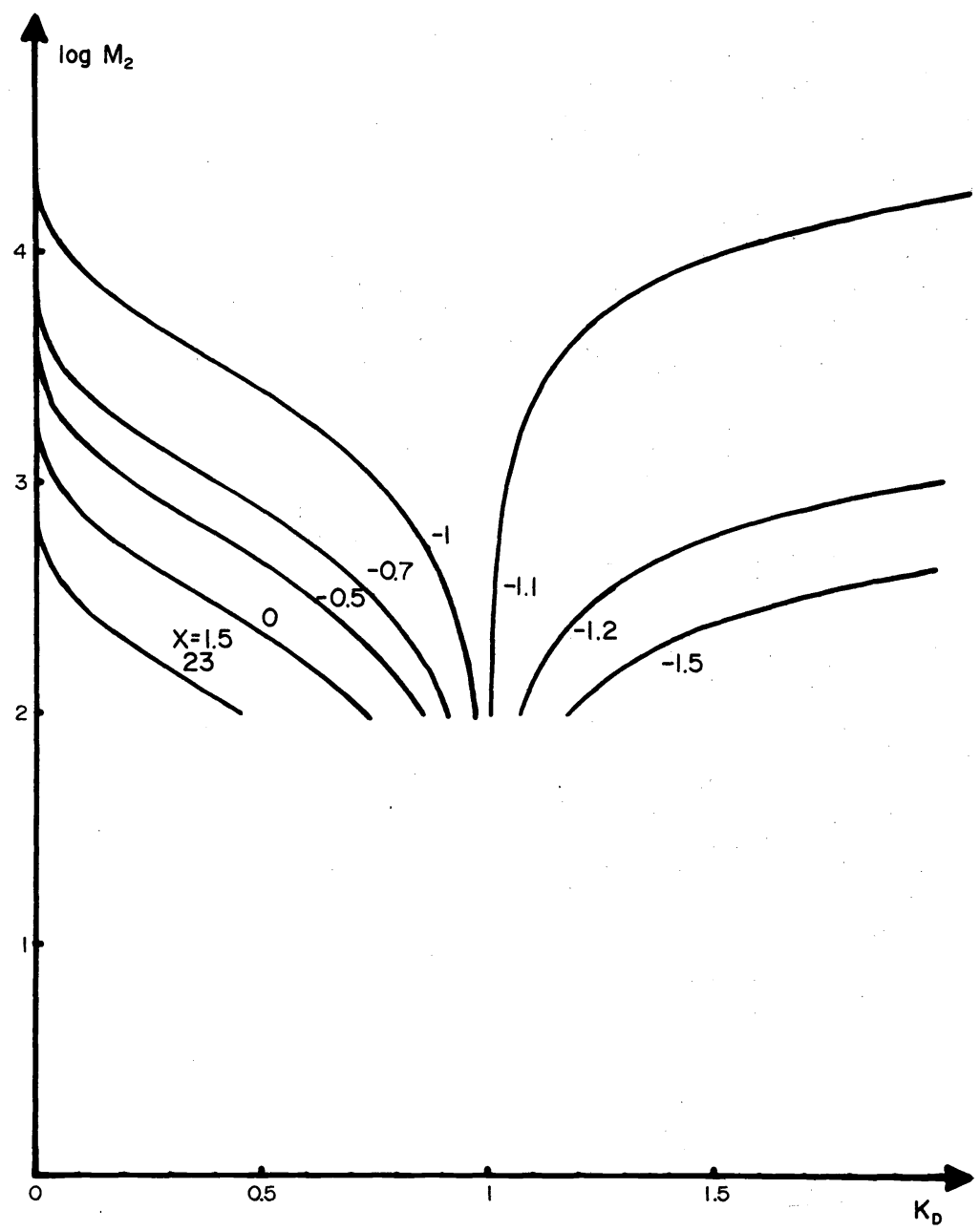

Fig. 7 Variation of $\log M_{2}$ versus $K_{D}$ for various values of $\chi_{23}$. Curves are plotted with $M_{2}=102 r_{2}, x_{3}=13$ and $x_{12}=x_{13}=0$.

As the affinity of the solute towards the gel increases ( $x_{23}$ decreasing), high molecular weights are more involved in the fractionation and, particalarly, when the solute/gel interaction is very strong, solutes can be therefore eluted in order of increasing molecular weights $\left(K_{D}>1\right)$. We observed this particular order of elution (Ref.31-32) with the triazinic gels described before, as shows in Fig. 8, where calibration curves for polystyrene and $n-a 1-$ kane samples in benzene and in dimethylformamide (DMF), are presented.

$K_{D}$ is higher for polystyrenes in DMF which is not such a good solvent like benzene for the same polymer. Conversely, n-alkanes are slightly soluble in DMF and their higher affinity for the gel makes their elutions in order of increased molecular weight. In that case, swollen gels really act as LLC stationary phases and by means of a "solvophobic effect" operate by reverse phase chromatography.

Similar results concerning that particular order of elution were published with other swollen gels as dextrane (Ref.36) and cellulose (Ref.37) and we have recently observed it (Ref.38) with common styrene/divinylbenzene packings, commercialized as Poragel $60 \AA$. Fig.9 shows that $n-a l c o h o l s$ and $n$-alkanes are eluted in DMF, in increasing molecular weights, beyond the $\left(V_{t}-V_{s}\right)$ volume. Conversely their elution volumes in THF remain in a classical order.

Accordingly, fractionation mechanism by selective dissolution of solutes into the gel phase, may be particularly important for swollen and lightly cross-linked gels, but it does not occur with rigid gels. The choice of the solvent nature as the gel nature, may minimize this non-exclusion effect in GPC. Beside, fractionation of low molecular weight compounds may be improved by taking into account the selective dissolution mechanism. 


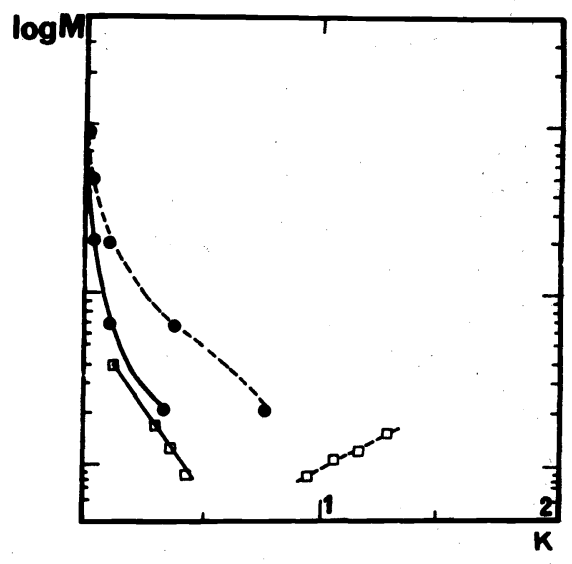

Fig. 8 Calibration curves of polystyrene ( $\bullet$ ) and $n$-alkanes ( $\square$ ) in benzene (-) and DMF (--) on a triazinic gel.

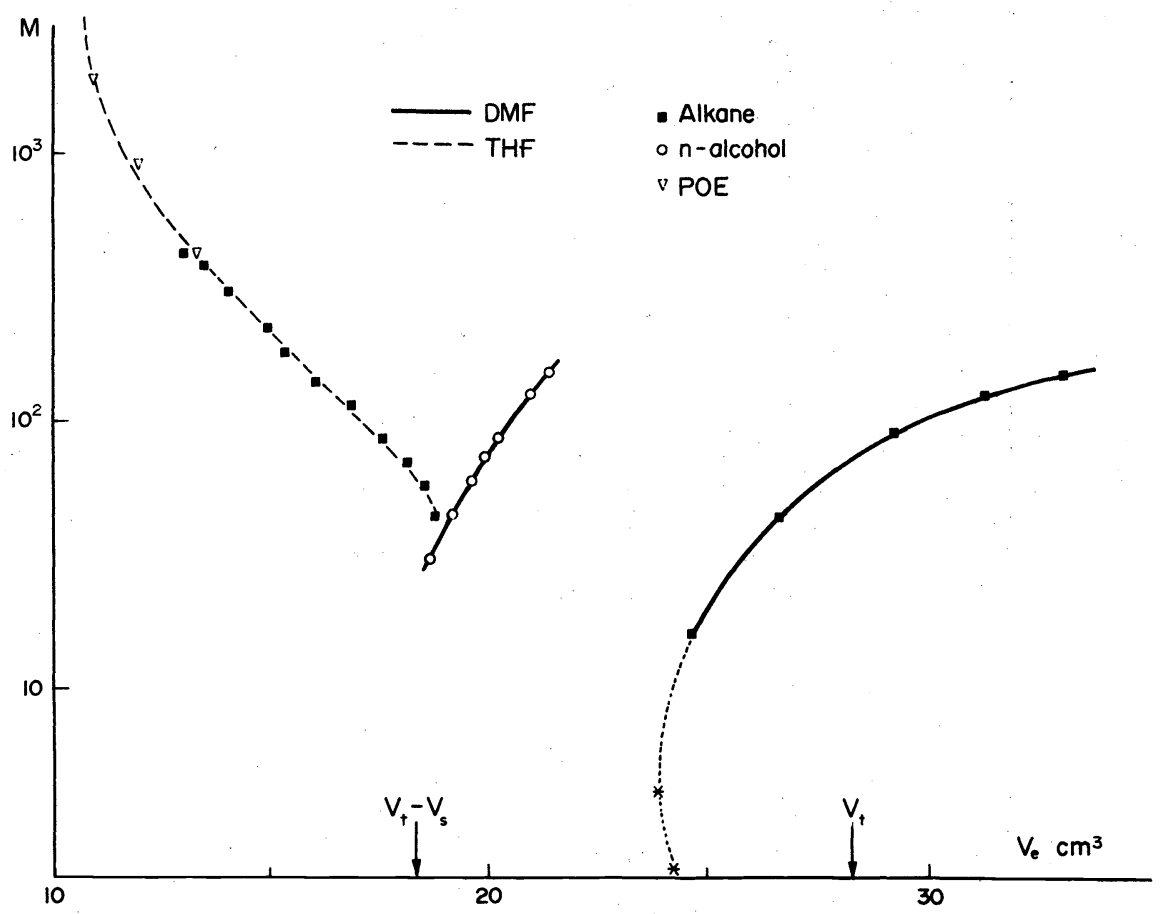

Fig.9 Calibration curves obtained with a styrene-divinylbenzene gel of low porosity (Poragel $60 \AA$ ).

\section{BONDED CHAIN STATIONARY PHASES}

Organic stationary phases, chemically bonded onto the surface of mineral supports such as silica, are widely used in LLC and have gained increasing importance during the past few years (Ref.5-11). Many experimental studies have been reported on the chromatographic behavior of these bonded phases, which structure was called "molecular fur" by Horvath and Melander (Ref.39). Most of them concern non-polar hydrocarbon-like bonded phases and several authors (Ref.40-45) pointed out the importance of the degree of surface coverage, since residual adsorption on these packings could greatly modify elution volumes. Besides, it was observed (Ref.46-47) that the longer the bonded carbon chain is, the stronger the retention of nonpolar solutes is. Several authors (Ref.48-50) showed that retention orders are, in general, function of the solute solubilities in the mobile phase. In order words, in reverse phase chromatography, molecular interactions between the solutes and stationary phase ("solvophobic interactions") play an important part in separations (Ref.51-52). 
Such experimental results bring contribution usefully to a best understanding of the nature of the different structural and molecular parameters involved in chromatographic retentions by such grafted phases but they have up to now poor theoretical bases, clearing the different mechanisms involved in LLC separations. Adsorption onto surface support, steric exclusion effect in the case of porous packings and partition by selective dissolution into grafted phase may be simultaneously occur.

Theoretical approach of the solute dissolution mechanism.

We have recently studied, in our laboratory, mechanisms of LLC separations performed on bonded phases, both in theoretical (Ref.53-54) and experimental (Ref.53,55-56) ways. Owing to our previous works on gels (Ref. 30 ), we first theoretically studied the partition mechanism of high molecular weight solutes between the mobile and stationary phases. In order to be in the most general case, we have considered that anchored grafts were constituted by non-entangling macromolecular chains of various molecular weights, chemically bonded onto a plane and inert surface, by one of their ends. That is, chains are independent and there is no adsorption of the solutes onto the wall (Ref.53-54).

As for gel stationary phases, the $K_{D}$ partition coefficient of a solute is given by :

$$
v_{e}=v_{0}+K_{D} \cdot v_{s}
$$

where $V_{S}$ is the volume of the swollen grafted phase.

The principle of the thermodynamical calculations we made (Ref.53-54), consists, by analogy with swollen gels, in evaluating the free energy changes $\Delta G$ of the solute/solvent and solute/ solvent/grafted phase mixtures, so as to equal the two expressions of the chemical potential of the solute in the two phases. Particularly, the free energy change of the solute/solvent/ grafted phase system is:

$$
\Delta G=\Delta G_{M}+\Delta G_{E L}
$$

where $\Delta G_{M}=\Delta H_{M}-T . \Delta S_{M}$ is the free energy change of mixing and $\Delta G_{E L}=-T . \Delta S_{E L}$, the elastic free energy due to the molecular expansion of the grafted chains iht the solveht.

Calculations of $\Delta H_{M}$ and $\Delta S_{F}$ terms were classically based on Flory's theory about regular macromolecular syslems. However, contrary to gel stationary phases, mathematical complicatiors arise, since the inert surface restricts the number of conformations taken by each macromolecular graft and by each macromolecular solute, being in its vicinity during the partition process. So as to evaluate the corresponding $\Delta S_{M}$ term, we made use of a calculation published by Casassa (Ref.57), which gives the fraction of the polymer chain conformations that still remains when one end of each chain is fixed at a given distance of a plane.

All these calculations lead to a rather elaborated expression of the partition coefficient $K_{D}(\operatorname{Ref} .53-54)$ :

$$
\begin{aligned}
& \log K_{D}=-r_{2}\left(\frac{1}{r_{1}}-\frac{1}{r_{3}}\right) \phi_{3}-\frac{\left(\phi_{3}^{1 / 3}-\phi_{3}\right)}{2} \frac{r_{2}}{r_{3}}+\log \left(1-\frac{r_{1}}{r_{3}} \phi_{3}\right) r_{2}+\chi_{12} \frac{r_{2}}{r_{1}} \phi_{3} \\
& +\chi_{13 \frac{r_{1}}{r_{1}} \phi_{1} \phi_{3}-\chi_{23} r_{2} \phi_{3}}-\frac{\phi_{3}^{4 / 3} \sqrt{\frac{2}{3 \pi} \frac{r_{1} r_{2}^{2}}{r_{3}^{3}}}}{\text { erf } \sqrt{\frac{3}{2} \frac{r_{1}}{r_{3}} \phi_{3}^{2 / 3}}}-\left(\frac{3}{2} \frac{r_{1}}{r_{3}} \phi_{3}^{2 / 3}\right) \\
& +\log \left[\operatorname{erf}\left(\phi_{3}^{-1 / 3} \sqrt{\frac{r_{3}}{r_{2}} \frac{3}{2}}\right)+\sqrt{\frac{2}{3 \pi} \frac{r_{2}}{r_{3}} \cdot \phi_{3}^{1 / 3}}\left(e^{\left.\left.-\left(\frac{3}{2} \frac{r_{3}}{r_{2}} \phi_{3}^{-2 / 3}\right)-1\right)\right]}\right.\right.
\end{aligned}
$$

where all the notations are the same as before (1: solvent ; 2 : solute and 3 : grafted phase).

The swelling of the grafted phase is expressed by the volume fraction $\phi_{3}$ of the swollen phase, which can be calculated by another equation : 


$$
\begin{aligned}
& \log \left(1-\phi_{3}\right)+\phi_{3}\left(1-\frac{r_{1}}{r_{3}}\right)-\log \left(1-\phi_{3} \frac{r_{1}}{r_{3}}\right)+\frac{r_{1}}{2 r_{3}}\left(\phi_{3}{ }^{1 / 3}-\phi_{3}\right) \\
& +\left(\operatorname{erf} \sqrt{\left.\frac{3}{2} \frac{r_{1}}{r_{3}} \phi_{3}{ }^{2 / 3}\right)^{-1}} e^{-\frac{3}{2} \frac{r_{1}}{r_{3}} \phi_{3}{ }^{2 / 3} \sqrt{\frac{2}{3 \pi}\left(\frac{r_{1}}{r_{3}}\right)^{3}} \phi_{3}{ }^{4 / 3}+x_{13} \phi_{3}{ }^{2}=0}\right.
\end{aligned}
$$

In Fig. 10, the swelling $\phi_{3}$ is plotted versus the solvent/phase interaction parameter $\chi_{13}$, for various values of the anchored chain size. We mention that the swelling of the grafted phase logically decreases with $x_{13}$ and increases with the molecular weight of the graft.

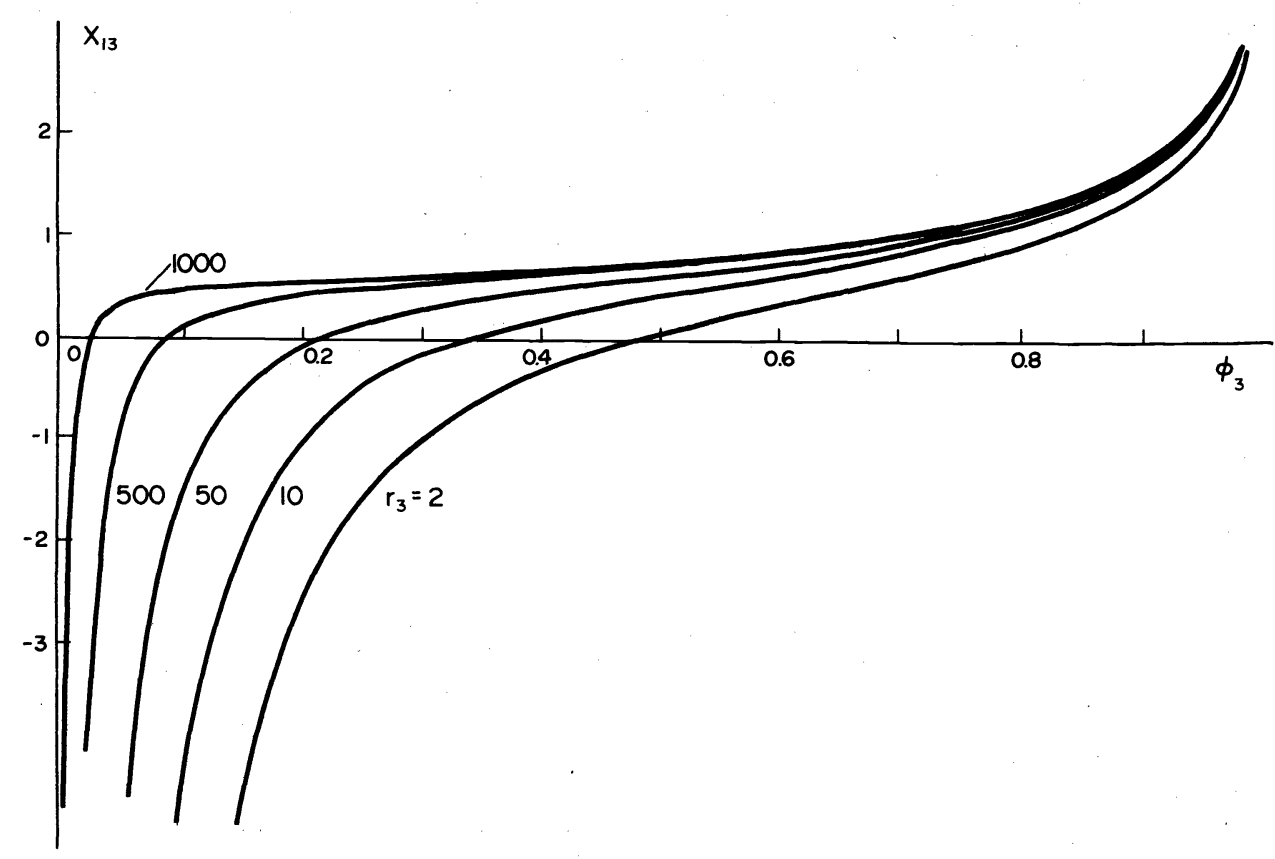

Fig.10 Swelling of the grafted phase $\left(\phi_{3}\right)$ versus $x_{13}$ for various values of
$r_{3}$.

As for gel stationary phases, the general expression of $K_{\mathrm{D}}$ allows to study the partition mechanism of solutes, according to their molecular weights expressed by $r$, and, particularly, the dependence of $K_{D}$ upon the molecular weight of the grafted chains $\left(r_{3}^{2}\right)$, the solvent nature $\left(x_{12}\right.$ and $\left.x_{13}\right)$ and the solute/grafted phase interaction $\left(x_{23}\right)$. Fig. 11 shows the calibration curbes $\log _{2}=f\left(K_{D}\right)$ obtained for various values of the $s$ ze parameter $r_{3}$ of the grafts, when there is no enthalpic effect $\left(x_{i j}=0\right)$.

Curves are similar to those corresponding to swollen gels: $K_{0}$ is changing form 0 to 1 , and the fractionation of high molecular weight solutes increases with the length of the grafted chains. As a result, an entropic effect may only cause a molecular weight fractionation. Besides, it can be showed (Ref. 53-54) that the solvent nature may alter these calibration curves. When one considers a series of solvents leading to identical swelling of the phase ( $x_{13} f i-$ xed) but having different affinities towards solutes, curves are systematically shifted upwards or downwards. For instance, the partition of high molecular weight solutes is more favoured when their interaction with the solvent is weak ( $x_{12}$ decreasing).

In another hand, it is interesting to notice the evolution of the calibration curves with the solute/grafts interaction parameter $\chi_{23}$, as shown in Fig. 12 ;

As the affinity of solutes towards the grafted phase increases ( $x_{23}$ decreasing), the fractionation concerns greater and greater values of solute molecular weights and when the solute/grafted chain interaction is rather strong, solutes are then eluted by increasing molecular weights $\left(K_{D}>1\right)$.

All these theoretical considerations demonstrate that swollen bonded stationary phases in 
their partition by dissolution mechanism, can be compared to swollen gels operating by the same process. Particularly, chromatographic behaviors of these two kinds of stationary phases are rather similar, when one substitutes the length between two cross-linked points of gels, by the length of grafted chains.

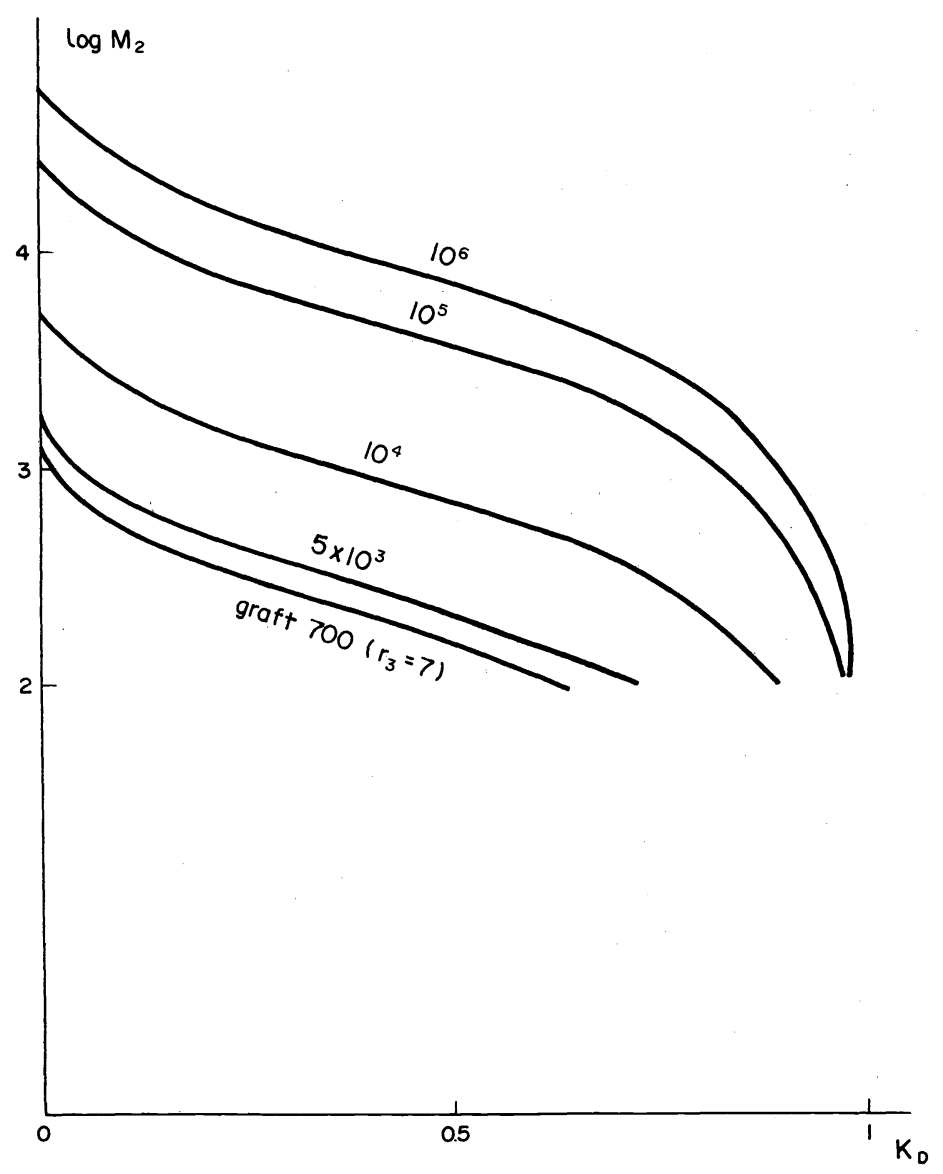

Fig. 11 Variation of $\log M_{2}$ (taken as $100 r_{2}$ ) versus $K_{0}$ for grafts of various molecular weight. Curves are plotted with $\chi_{12} \stackrel{D}{=} \chi_{13}=\chi_{23}=0$.

Experimental study of silica/polyethyleneoxide bonded phases.

As there is no well-defined commercial macromolecular bonded phases, we have recently prepared some grafted silica packings, by bonding macromolecular chains of various chemical natures and molecular weights (Ref.55-56). These stationary phases, which can be considered as model bonded phases, allowed us to study systematically the mechanisms involved in the observed chromatographic characteristics.

We prepared first silica/polyethyleneoxide phases by heat treating $40 / 60$ um porous silica "Si 60 Merck" with pure polyethyleneoxide samples of different molecular weights: 2.102 , $4.10^{2}, 2.10^{4}$ and $5.10^{6}$. Table 1 gives specific surface area and porous volume data, determined by nitrogen adsorption. Silica loading $\tau$, which is defined by the ratio of the weight of bonded polyethyleneoxide to the overall weight of the final packing, was measured by ther mogravimetry and elemental analysis.

These bonded phases do not apparently swell, even in good solvents of the grafts and the magnitude order of the porous volume decreasing with increasing loading values, indicates that polyethyleneoxide chains are mainly grafted inside silica pores, as we will show later (see Fig. 16).

Fig. 13 shows typical calibration curves obtained by eluting polyethyleneoxide samples in one of their poor solvents, such as acetonitrile, on silica-PEO 400.

As we could expect it, polyethyleneoxide solutes are very adsorbed on pure silica and are therefore eluted in increasing molecular weights. In the case of bonded phases, the elution 
order is a function of the silica loading : adsorption also occurs but for an adequate amount of bonded stationary phase, an inversion of the solute elution order is observed. Critical values $\tau$ corresponding to all these bonded phases, except for silica-PEO 200, are always about $13=15 \%$.

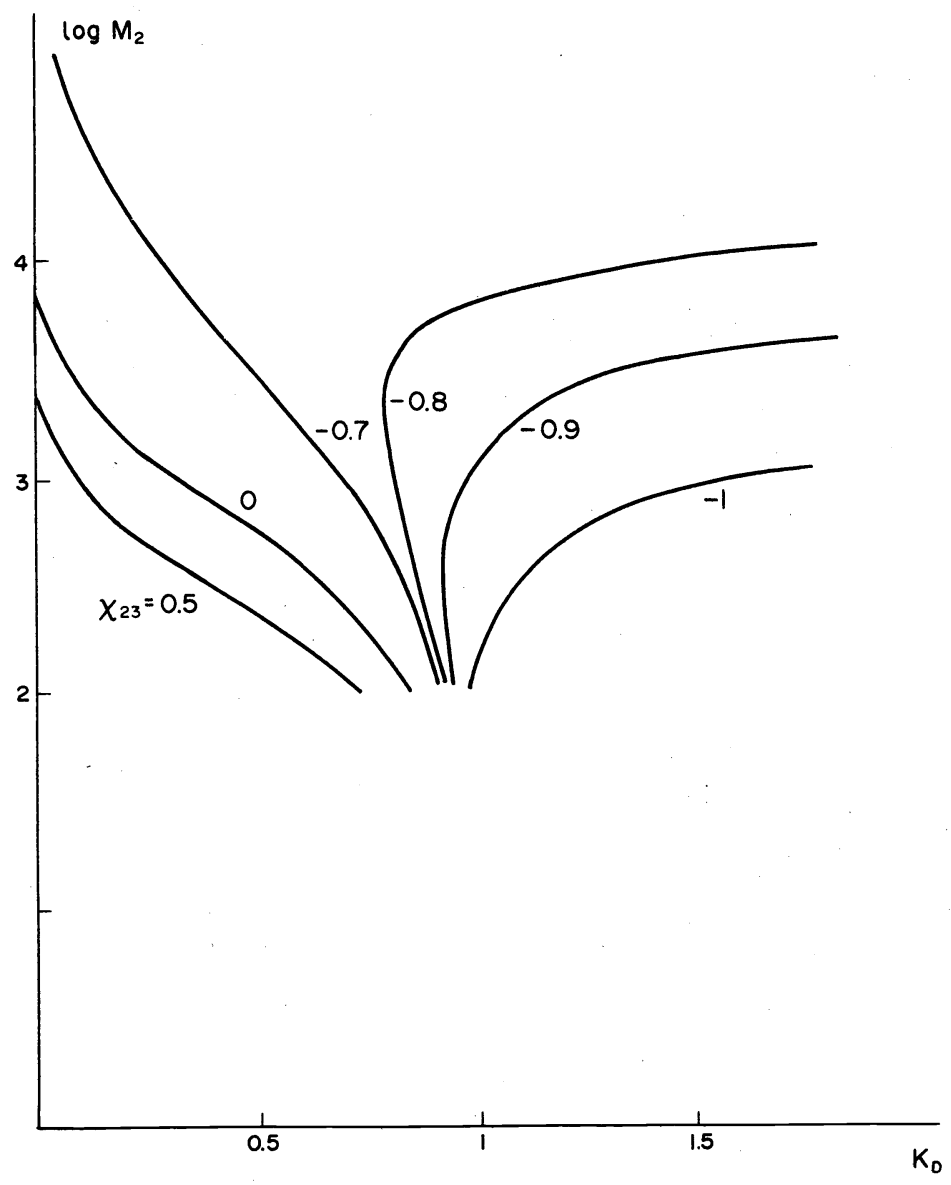

Fig. 12 Variation of $\log M_{2}\left(M_{2}\right.$ taken as $\left.100 r_{2}\right)$ versus $K_{0}$ (partition coefficient) for various values of $x_{23}$. Curves are plotted with $r_{3}=10$ and $x_{12}=x_{13}=0$.

\begin{tabular}{|l|c|c|c|}
\hline Bonded phase & $\begin{array}{c}\mathrm{S} \\
\left(\mathrm{m}^{2} / \mathrm{g}\right)\end{array}$ & $\begin{array}{c}\mathrm{V}_{\mathrm{p}} \\
\left(\mathrm{cm}^{3} / \mathrm{g}\right)\end{array}$ & $\tau$ \\
\hline Si 60 & & & \\
Si 60-(PE0 200) & 300 & 0,50 & $12 \%$ \\
Si 60-(PEO 400) & 240 & 0,50 & $14 \%$ \\
Si 60-(PE0 2.10 & & \\
Si 60-(PE0 5.106) & 200 & 0,40 & $22 \%$ \\
& 290 & 0,52 & $13 \%$ \\
\hline
\end{tabular}

TABLE 1. Specific surface area, porous volume and loading values of silica/polyethyleneoxide bonded phases. 


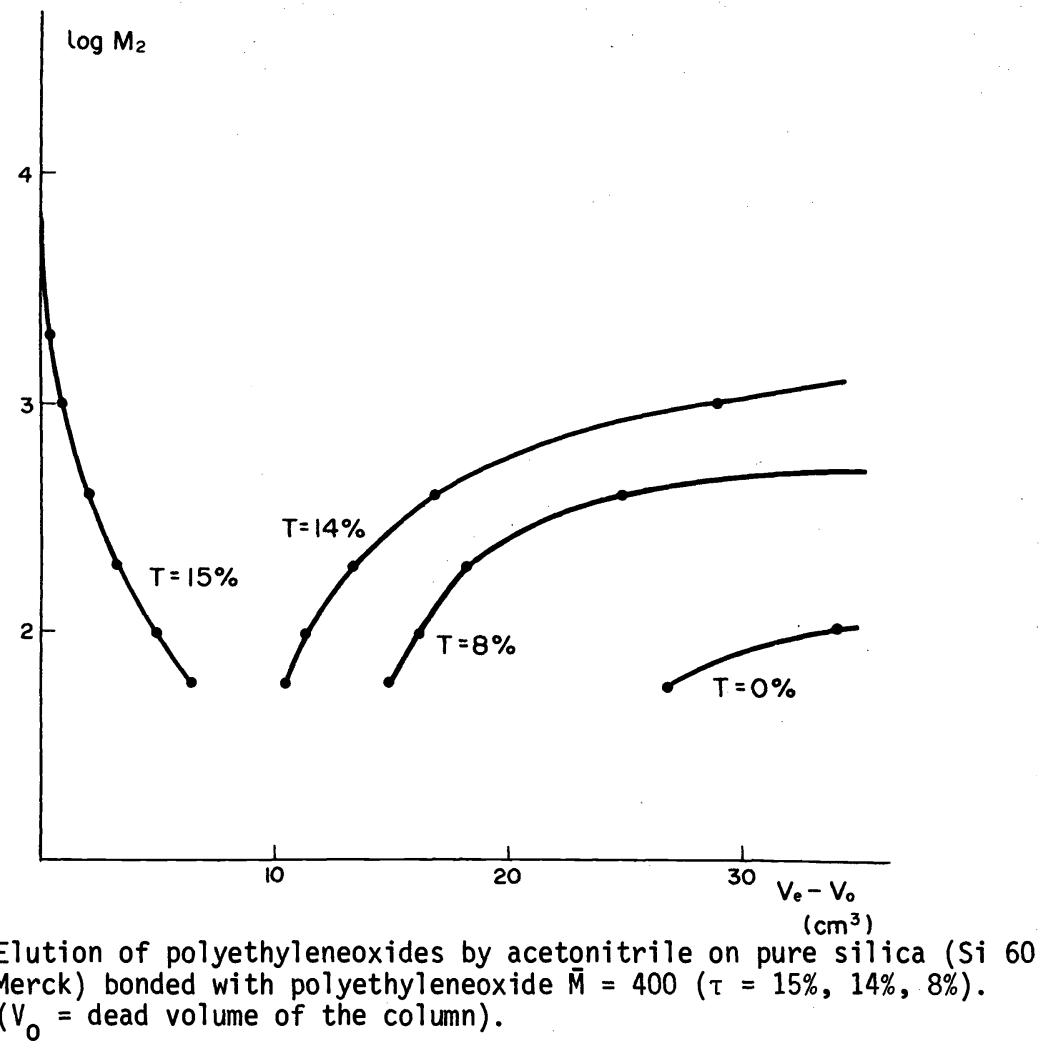

We have given a molecular interpretation to that notion of critical loading $\tau$, assuming that the surface area concealed by each macromolecular graft, in a poor solvent, is taken approximately as the great circle surface of its own swollen volume. In that way, it can be deduced that the total surface of silica concealed by all grafted chains, is proportional to $\tau$ and does not depend on the graft molecular weight. Then, it appears that the critical loading value $\tau_{c}=13-15 \%$ may correspond to the concealment of the overall surface.

As a result, for $\tau<\tau$, steric exclusion effect and partition by dissolution occur, but adsorption predominate $\xi^{\prime}$ and when $\tau>\tau$, partition by dissolution into the grafted phase becomes the main mechanism. However, in the particular case of silica bonded with short grafts, such as PEO 200, even if the silanol groups of silica were totally substituted, the critical value $\tau$ would not be reached. One actually observes, for that packing, that retentionsalways increase with solute molecular weights, for all the loading levels studied. All these results indicate that for chromatographic separations based on adsorption mechanism, retention volumes are mainly function of the graft molecular weights and of the degree of surface coverage. Besides, it is possible to modify the chromatographic behavior of these bonded phases by varying these two parameters.

A more interesting aspect concerns experiments where the solvent used is a good one for solutes and therefore adsorption is not predominant, even on pure silica. Fig. 14 gives typical calibration curves obtained by eluting polyethyleneoxide samples in DMF.

The curve corresponding to pure silica is a classical GPC one. On grafted phases, elution volumes are smaller than on pure silica, whatever the molecular weight of the grafts could be. However, the elution volyme of a given solute decreases when the graft molecular weight increases form 2.102 to $2.10^{4}$, but increases for the ultimate value of 5.106. The same holds true for other solute/solvent systems, such as n-alkanes in THF or polyisobutene in THF (see Fig. 15).

An explanation of that particular chromatographic behavior can be proposed from the model of a silica/polyethyleneoxide bonded phase, shown in Fig.16. The "molecular fur" picture is a good one here, as grafted chains are mainly bonded inside silica pores. As a result, the solute fractionation proceeds by a steric exclusion mechanism into the residual porous volume (zone II) and then, by a dissolution mechanism into the grafted phase (zone I).

We could evaluate the general expression of solute elution volumes, in terms of these two consecutive mechanisms (Ref.55) :

$$
v_{e}=v_{0}+K_{G P C}^{1} \cdot K_{D} \cdot v_{p}+\left(1-K_{D}\right) \cdot K_{G P C}^{2} \cdot\left(V_{p}-V_{s}\right)=v_{0}+\bar{K} \cdot v_{p}
$$


where $K_{D}$ is the partition coefficient by selective dissolution into the grafted phase volume $V_{s} ; K_{G P C}$, the GPC partition coefficient in the overall porous volume $V_{p}$ and $K_{G P C}$, the GPC partitiff coefficient in the residual porous volume $\left(v_{p}-v_{s}\right)$.

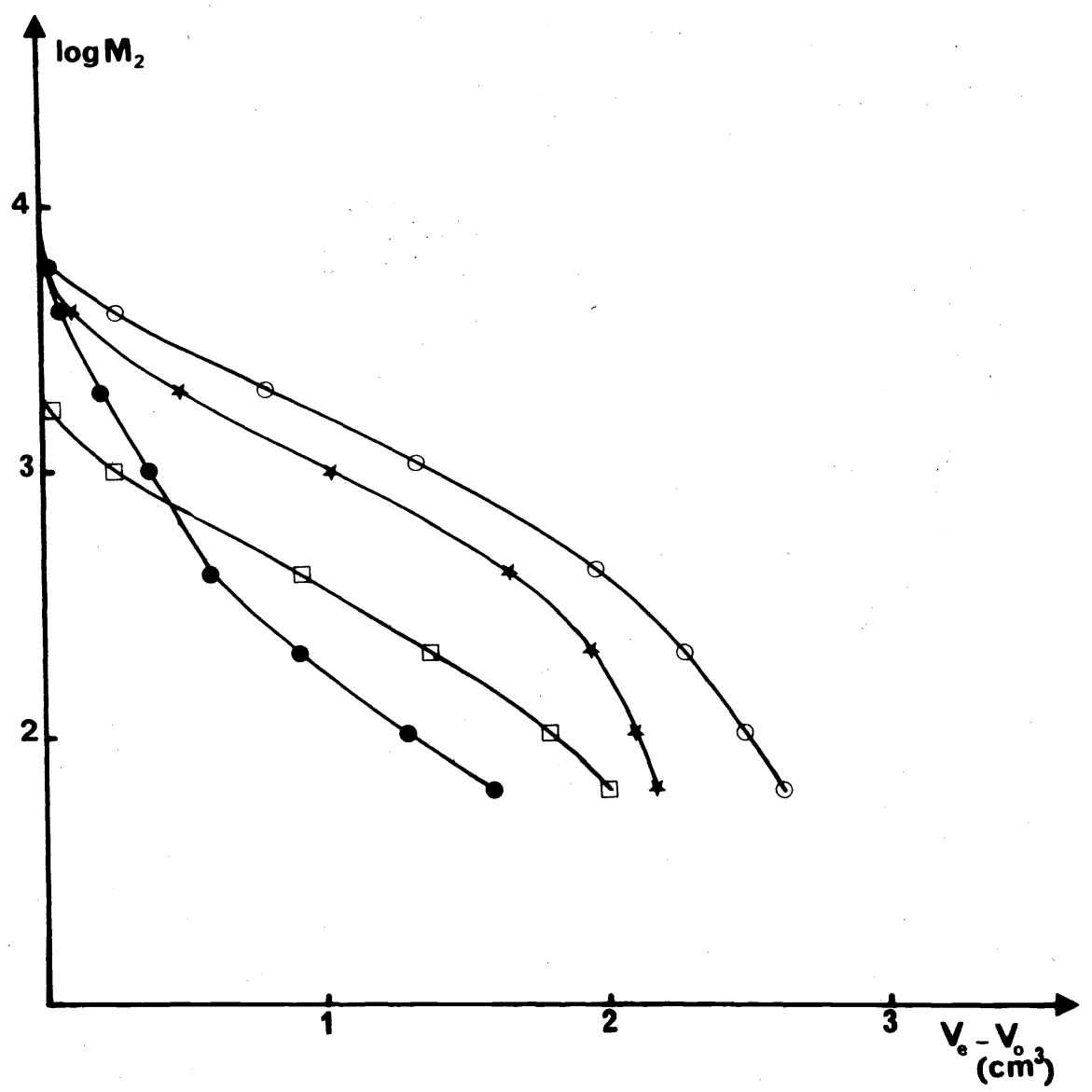

Fig. 14 Elution of polyethyleneoxides by dimethylformamide on pure silica $(O)$ and silica bonded with polyethyleneoxide $\bar{M}=200(\star)$, $\vec{M}=5.106(\square)$ and $\bar{M}=2.10^{4}(\square)$. $K$ was calculated from the theoretical equations we gave before and $K_{G \mathrm{PC}}^{1}$ and $\mathrm{K}_{\mathrm{GPG}}^{2}$ were
roughily evaluated from the characteristics of pure and grafted silicas. ${ }_{\mathrm{Fig}} 17_{\mathrm{Gg}} \mathrm{g}$ ves the theoretical cal ibration curves $\log M_{2}=f(\bar{K})$, for the molecular weight values of the grafts used and by taking $x_{i j}=0$. These curves are qualitatively in good agreement with our experiments : residual steric exclusion effect and selective dissolution effect are then opposed. By increasing the graft molecular weight, elution volumes decrease by GPC effect with pore size decreasing, and after increase for higher molecular weight grafted chains, by a predominant dissolution mechanism.

The main consequence of the experimental study we have carried out on these silica/polyethyleneoxide bonded phases (Ref:53-55), concerns the grafted GPC packings commercialized for the molecular weight characterization of organo or hydrosoluble polymers (Ref.12-16). Particularly recent " $\mu$-bondagel" packings (Ref.15-16) have polyether grafts similar to our model bonded phases. In that case, the experimental results we obtained indicate that such grafts located into pores, would appreciably alter GPC calibration curves, especially for packing of low porosity range.

Experimental study of silica/polystyrene bonded phases.

As non-polar bonded phases are frequently used in a wide range of separation problems, especially in reverse phase chromatography, we have studied, in a parallel way, silica/polystyrene bonded phases with various molecular weight grafts (Ref.53-56).

These model bonded phases were prepared by deactivation of living anionic polystyrene samples in benzene, on chlorinated silica, at $0^{\circ} \mathrm{C}$, following the reaction : 

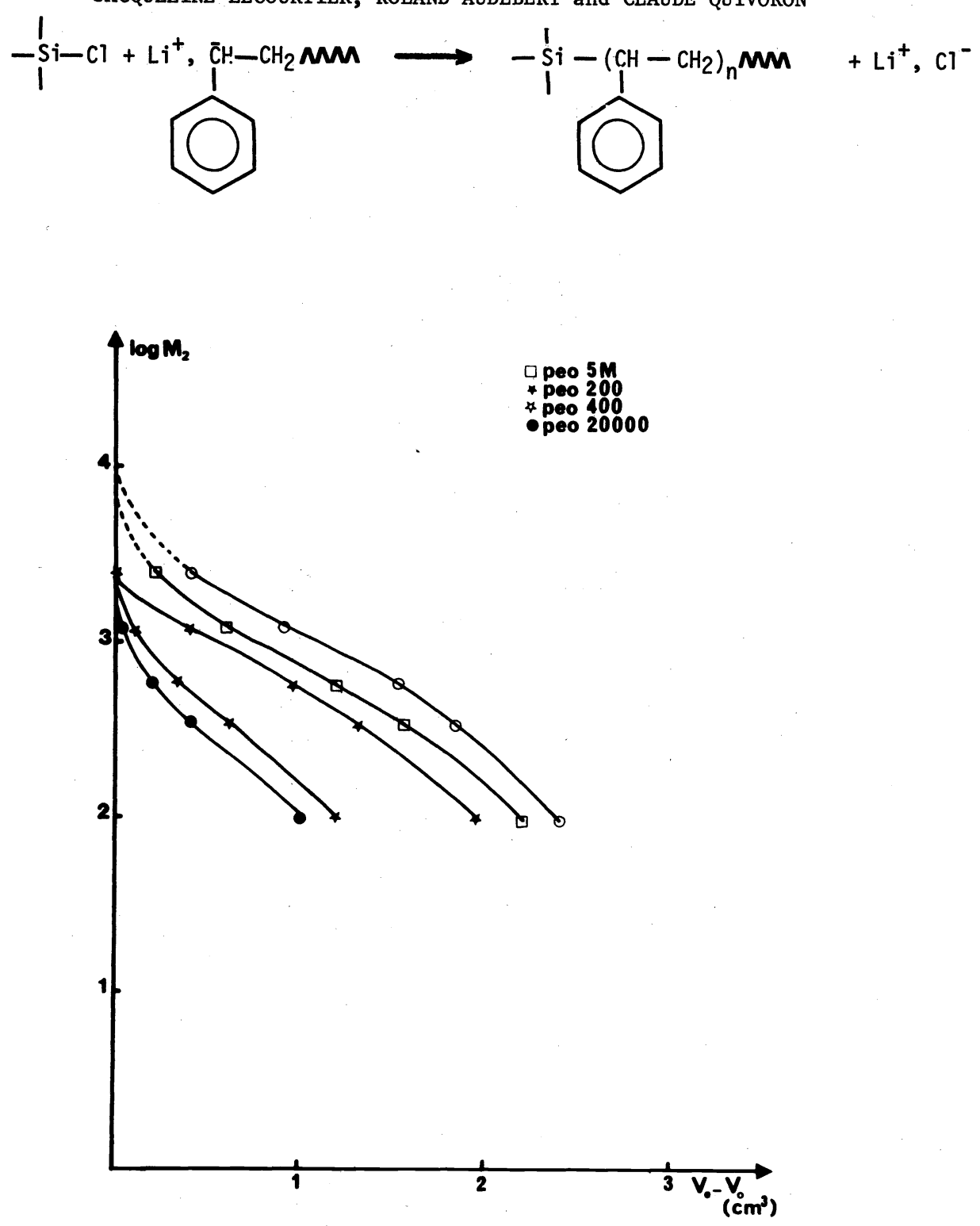

Fig. 15 Elution of polyisobutene by THF on pure silica ( 0 ) and silica bonded with polyethyleneoxides of various molecular weight.

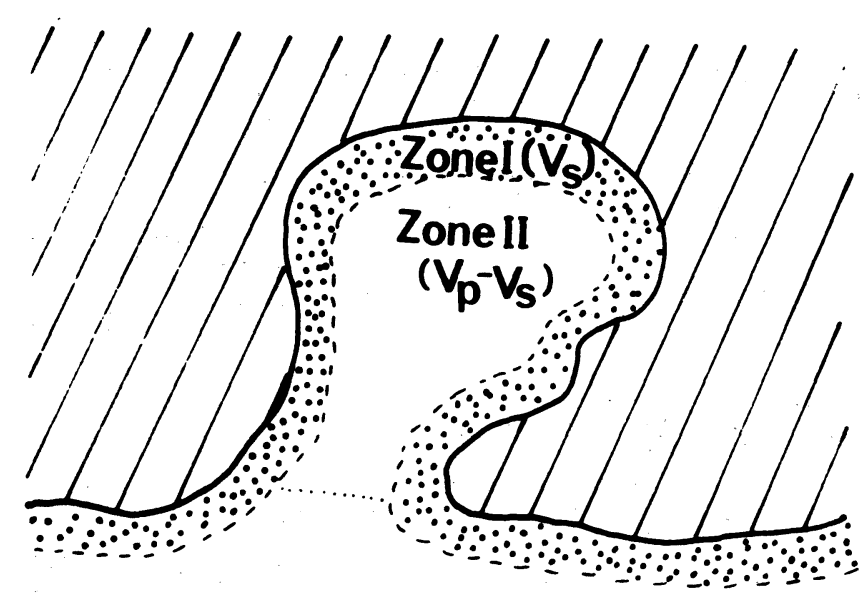

Fig. 16 Model of silica - polyethyleneoxide packing 


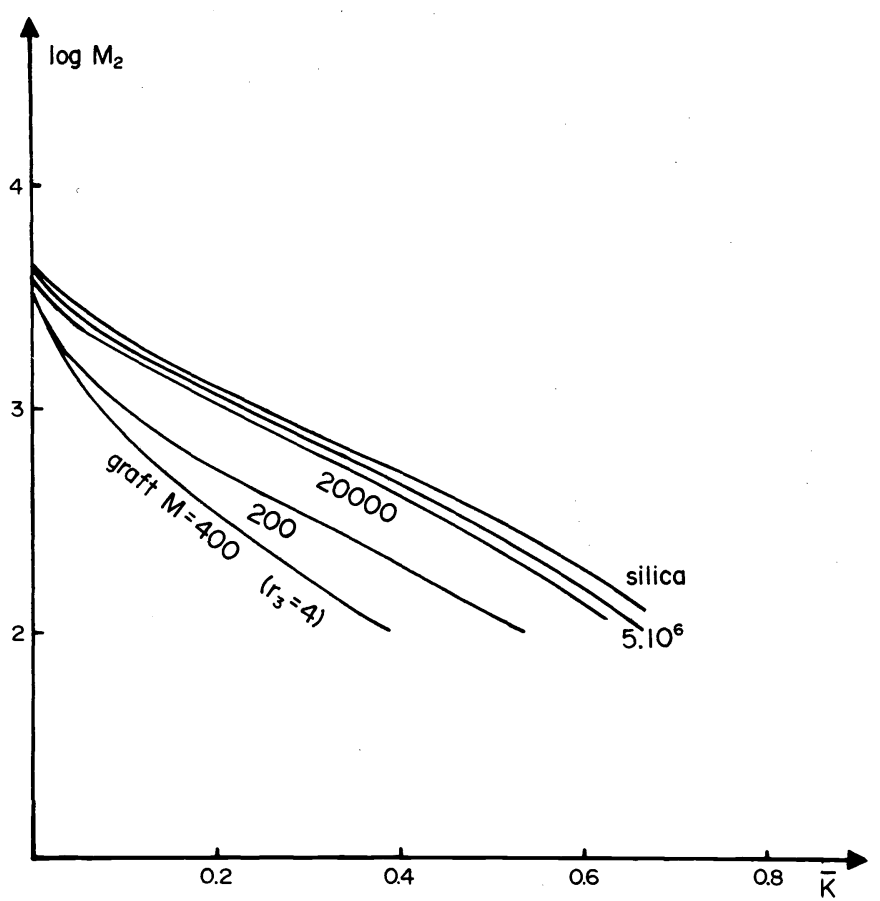

Fig. 17 Calculated calibration curves for silica and silica bonded with polyethyleneoxides of various molecular weight.

Bonded polystyrene chains were assumed to have the same molecular weight as chains which did not react with silica and whose molecular weights were determined by GPC : 1000,8000 and 50 000. Table 2 gives specific surface area, porous volume and loading values of each packing.

\begin{tabular}{|c|c|c|c|}
\hline Bonded phase & $\left(m^{2} / g\right)$ & $\left(\mathrm{cm}^{3} / \mathrm{g}\right)$ & $\tau$ \\
\hline Si 60 & 345 & 0,58 & 0 \\
\hline Si $60-(P S 1000)$ & 60 & 0,15 & $8 \%$ \\
\hline Si $60-($ PS 8000$)$ & 50 & 0,09 & $10 \%$ \\
\hline Si $60-($ PS 50000$)$ & 145 & 0,37 & $5 \%$ \\
\hline
\end{tabular}

TABLE 2. Specific surface area, porous volume and loading values of silica/polystyrene bonded phases.

Contrary to silica/polyethyleneoxide phases, one observes an appreciable variation of the porous volume with silica loading (amazingly, lg of grafted polymer leads to a decrease of about $5 \mathrm{~cm}^{3}$ ). Besides, the swelling of these bonded phases in good solvents (chloroform or THF) of polystyrene, can reach about $10 \%$ in volume. All this suggests that polystyrene grafts are mainly bonded outside silica pores. In this way, during nitrogen adsorption experiments, at $-195^{\circ} \mathrm{C}$, grafted chains prevent nitrogen molecules from penetrating into the pores; which explain the $S$ and $V_{p}$ values obtained.

As for silica/polyethyleneoxide bonded phases, we have studied solute/solvent system which lead or not to adsorption.

Fig. 18 gives, for instance, calibration curves corresponding to polystyrene solutes in decalin. In that poor solvent, adsorption occurs on pure silica and samples are eluted in increasing molecular weights. For silica bonded with PS 1000 , elution volumes slightly decrease with the solute size, but for grafts PS 8000 and 50000 , retention volumes increase with solute molecular weights. Particularly, retention is stronger on silica bonded with PS 50000 than on pure silica. Similar results were obtained with polyethyleneoxide samples in methanol. Qualitatively, the three mechanisms : adsorption on silica, steric exclusion in 
silica pores and selective dissolution into the grafted phase, may simultaneously occur. Our theoretical study of partition coefficient $K_{0}$ (Ref. 53-54) showed that for solutes dissolved in poor solvent, $K_{D}$ may be smaller than one for short grafts (such as PS 1000 ) and converse$1 y$ may be greater than one, for higher values of graft molecular weights (such as 8000 and 50 000). This particularly explains why retention of polystyrene samples in decalin, is stronger on PS 50000 packing than on pure silica.

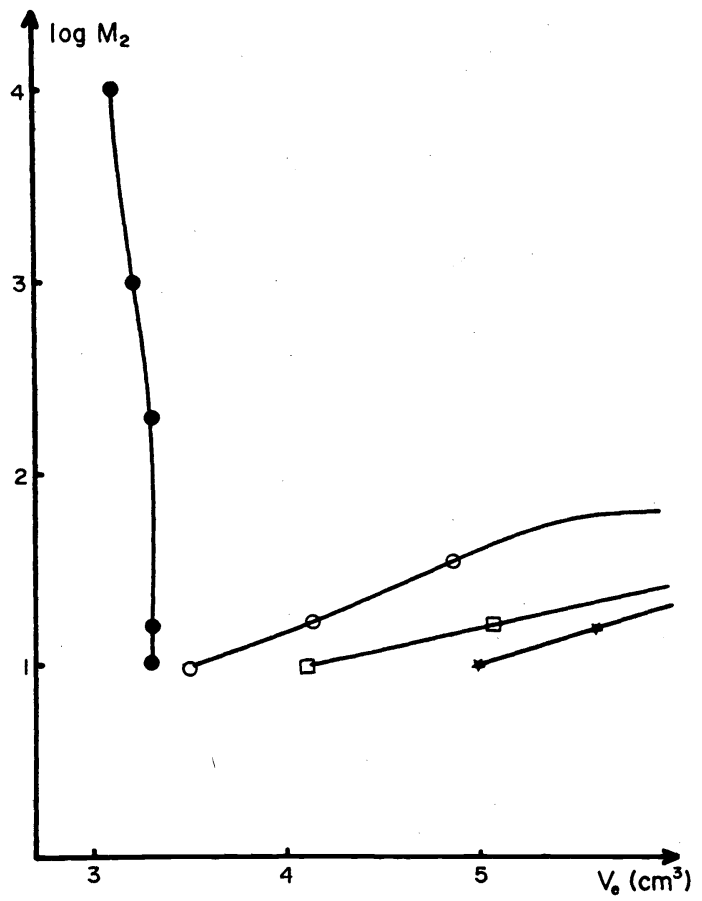

Fig. 18 Elution of polystyrene solutes by decalin on pure silica ( $\square$ ) and silica bonded with polystyrenes $M=1000$ ( $\bullet), M=8000$ ( 0 ) and $M=50000(\star)$.

Fig. 19 shows typical calibration curves when there is no adsorption effect on pure silica, for instance in the case of polystyrene samples in chloroform. One observes that exclusion limit and elution volumes on grafted phases, increase gradually as the graft molecular weight increases. Besides, contrary to silica/polyethyleneoxide bonded phases, silica/polystyrene ones can become more efficient than pure silica, for high molecular weight fractionations.

We have proposed an interpretation based on the fact that polystyrene grafts are mainly bonded outside silica pores, as shown in Fig.20. The bonded chains prevent the direct access to the pores and solutes have to be dissolved in a first time, into the grafted phase, before penetrating into the porous volume. The mechanistic process is here reverse to the operating one for silica/polyethyleneoxide bonded phases. We could calculate the general expression of solute elution volumes, in terms of these two consecutive equilibria:

$$
v_{e}=V_{0}+K_{D} \cdot v_{s}+K_{D} \cdot K_{G P C} \cdot v_{p}
$$

where $K_{D}$ is the partition coefficient by selective dissolution into the grafted phase volume $V_{s}$; $K_{G P C}$, the GPC partition coefficient in the overall porous volume $V_{p}$ of pure silica.

We have evaluated $K_{0}$ from our theoretical expressions, for grafts with molecular weight 1000 , 8000 and 50000 . FPg.21 gives the theoretical calibration curves obtained for each molecular weight and by taking $x_{i j}=0$. There is a good agreement with the experimental curves we obtained. Particularly, $j_{i t}$ is interesting to notice that above the exclusion limit of pure silica (4000), there is no more exclusion steric effect and fractionations are only based on the selective dissolution mechanism, which is then more important as the graft molecular weight increases.

Comparison between silicas bonded with polyethyleneoxide or with polystyrene, emphazises the importance of the grafting method, which leads to grafts located either inside or outside the silica pores and the chromatographic behavior of these packings cannot be elucidated if this difference is neglected. Besides, for separations of low or high molecular weight solutes 
on chemically bonded phases, for which the dissolution mechanism into the grafted stationary phase is more operative than the adsorption one, it may be interesting to graft high molecular weight chains and to use high loading, in spite of the decrease in efficiency which would result.

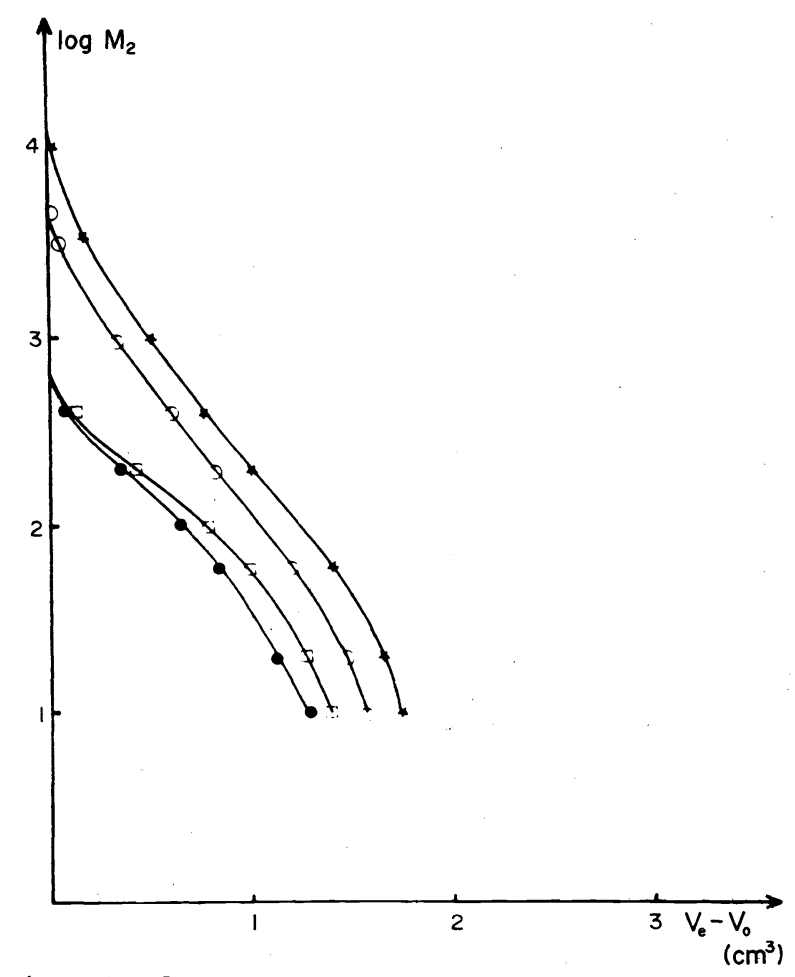

Fig. 19 Elution of polystyrene solutes in chloroform on pure silica ( $\square)$ and silica bonded with polystyrenes $M=1000(\bullet), M=8000$ (O) and $M=50000(\star)$.

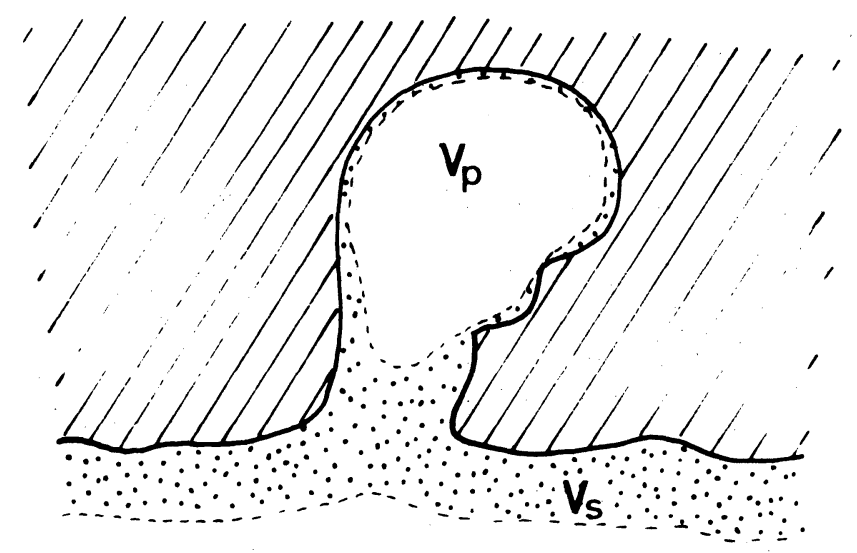

Fig. 20 Model of silica - polystyrene packing. 


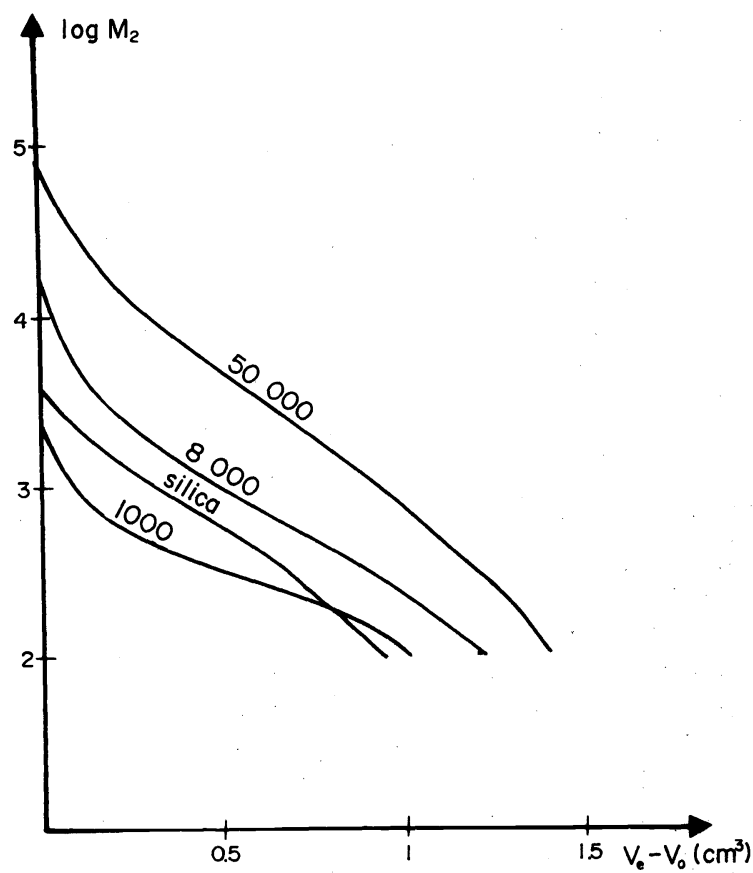

Fig. 21 Calculated calibration curves for silica and silica bonded with polystyrene of various molecular weight.

\section{REFERENCES}

1. K. ALGELT and L. SEGAL, Gel Permeation Chromatography, Marcel Dekker, New York (1971).

2. V. GAYLOR and H. JAMES, Anal. Chem., 50, 29R (1978).

3. S. POKORNY, Chem. Listy, 68,1088 (1974).

4. M. MINARIK and R. KOMERS, Chem. Listy, 68, 696 (1974).

5. H. WALTON, Anal. Chem., 50, 36 R (1978).

6. E. GRUSHKA, Bonded Stationary Phases in Chromatography, Ann Arbor Sci. Michigan (1974).

7. D. C. LOCKE, J. Chromatog. SCi., 11, 120 (1973).

8. R. MAJORS, Analusis, 3,549 (1975).

9. A. PRYDE, J. Chromatog. Sci., 12,486 (1974).

10. V. REHAK and E. SMOLKOVA, Chromatographia, 9 (5), 219 (1976).

11. E. GRUSHKA and E. KIKTA, Anal. Chem., 49, 1004 A (1977).

12. S. H. CHANG, K. M. GOODING and F. E. REGNIER, J. Chromatog., 120, 321 (1976); 125, $103(1976)$.

13. S. H. CHANG, R. NOEL and F. E. REGNIER, Analytical Chem., 48, 1839 (1976).

14. C. PERSIANI, P. CUKOR and K. FRENCH, J. Chromatog. SCi., 14, 417 (1976).

15. Waters Associates, $\mu$-bondagel packing, Milford, U.S.A.

16. R. VIVILECCHIA, B. LIGHTBOLY, N. THIMOT and H. QUINN, J. Chromatog. Sci., 15, 424 (1977).

17. R. AUDEBERT, Analusis, 4 (9), 399 (1976).

18. B. G. BELENKII, L. Z. VILENCHIK, V. V. NESTEROV, V. J. KOLEGOV and S. Y. FRENKEL, J. Chromatog. , 109, 233 (1975).

19. J. V. DAWKINS and M. HEMMING, Makromol. Chem., 176, 1777 (1975); 176, 1795 (1975); 176, 1815 (1975)

20. J. V. DAWKINS, J. Polym. Sci., Polym. Phys. Ed., 14, 569 (1976); J. Liquid Chrom., 1 (3), 279 (1978)

21. M. R. AMBLER, J. Polym. Sci., Polym. Lett. Ed., 14, 683 (1976).

22. P. L. DUBIN, Ind. Res., $18(7), 55$ (1976).

23. P. L. DUBIN, S. KOONTS and K. L. WRIGHT, J. Polym. Sci., Polym. Chem. Ed. , 15, 2047 (1977).

24. D. H. FREEMAN and D. KILLION, J. Polym. Sci., PoTym. Phys. Ed., 15, 2047 (1977).

25. M. MINARIK and J. COUPEK, MakromoT. Chem., 64, 147 (1977).

26. B. G. BELENKII, E. S. GANKINA, M. B. TENNIKOV and L. A. VILENCHIK, J. Chromatog., 147, 99 (1978).

27. D. BEREK, D. BAKOS, T. BLEHA and L. SOLTES, Makromol. Chem., 176, 391 (1975).

28. W. KISSING, R. REINER, Chromatographia, 11, 83 (1978).

29. Z. GRUBISIC, P. REMPP and BENOIT, J. Polym. Sci., Part B, 5,753 (1967).

30. J. LECOURTIER, R. AUDEBERT and C. QUUIVORON, J. Chromatog. , 121, 173 (1976).

31. M. C. MILLOT, J. LESEC, R. AUDEBERT and C. QUIVORON, Analusis, 4 (9), 410 (1976).

32. J. LECOURTIER, R. AUDEBERT et C. QUIVORON, Chromatography of Synthetic and Biological Polymers, Ellis Horwood Ltd, Chichester, West Sussex, Vol.1 (Chap.9), 156 (1978). 
33. P. WEISS, J. HERZ, P. REMPP, Z. GALLOT and H. BENOIT, Makromol. Chem., 145, 105 (1971).

34. G. HILD, D. FROELICH, P. REMPP and H. BENOIT, Makromol. Chem., 151, 59 (1972).

35. Y. MOTOZATO, N. KUSUMOTO, C. HIRAYAMA, R. MURAKAMI and H. ISOZAKI, Polymer, 16, 321 (1975).

36. N. MARSDEN, Ann. N. Y. Acad. Sci., 125, 428 (1965).

37. K. CHITUMBO and W. BROWN, J. Chromatog. , 80, 187 (1973).

38. R. AUDEBERT, 6ème Colloque National de GPC, Grenoble, July $3-4$ ( 1978 ).

39. C. HORVATH and W. MELANDER, J. Chromatog. Sci. , 15, 393 (1977).

40. J. N. LITTLE, W. A. DARK, P. W. FARLINGER and K. BOMBAUGH, J. Chromatog. Sci., 8,647 (1970).

41. E. GRUSHKA and E. J. KIKTA, Anal. Chem., 48, 1098 (1976).

42. I. HALASZ and J. SEBASTIAN, J. Chromatog. Sci., 12, 161 (1974).

43. J. KIRKLAND, J. Chromatog. Sci., 9, 206 (1971)'; Anal. Chem., 43, 36 (1971).

44. F. K. UNGER, N. BECKER and P. ROOMELIOTIS, J. Chromatog. , 125, T15 (1976).

45. M. MINARIK, R. KOMERS and J. COUPEK, J. Chromatog., 148, 175 (1978).

46. H. HEMETSBERGER, W. MAASFIELD and H. RICKEN, Chromatographia, 9, 303 (1976).

47. R. K. GILPIN, J. A. KORPI and G. A. JANICKI, AnaT. Chem., 47, I498 (1975).

48. D. C. LOCKE, J. Chromatog. Sci., 12, 433 (1974).

49. W. SCOTT and P. KUCERA, J. Chromatog., 112, 425 (1975).

50. E. GRUSHKA and E. J. KIKTA, Anal. Chem. , 46, 1370 (1974).

51. B. L. KARGER, J. R. GRANT, A. HARTKOPP and P. H. WEINER, J. Chromatog., 128, 65 (1976).

52. C. HORVATH, W. MELANDER and I. MOLNAR, J. Chromatog., 125, 129 (1976).

53. J. LECOURTIER, Thesis, Paris (1977).

54. J. LECOURTIER, R. AUDEBERT and C. QUIVORON, to be published in Macromolecules.

55. J. LECOURTIER, R. AUDEBERT and C. QUIVORON, J. Liquid Chrom., 1 (3), 367 (1978).

56. J. LECOURTIER, R. AUDEBERT and C. QUIVORON, J. Liquid Chrom. , I (4), 479 (1978).

57. E. F. CASASSA, Separation Science, 6 (2), 305 (1971). 